\title{
XIPE the X-ray Imaging Polarimetry Explorer
}

Soffitta P. ${ }^{1}$, Bellazzini R. ${ }^{2}$, Bozzo E. ${ }^{3}$, Burwitz V.4 , Castro-Tirado A. J. ${ }^{5}$, Costa E. ${ }^{1}$, Courvoisier T. ${ }^{3}$, Feng H. ${ }^{6,7}$, Gburek S. ${ }^{8}$, Goosmann R. ${ }^{9}$, Karas V. ${ }^{10}$, Matt G. ${ }^{11}$, Muleri F. ${ }^{1}$, Nandra K.4, Pearce M.43, Poutanen J. ${ }^{12}$, Reglero V. ${ }^{16}$, Sabau Maria D. ${ }^{13}$, Santangelo A. ${ }^{14}$, Tagliaferri G. ${ }^{15}$, Tenzer C. ${ }^{14}$, Vink J. ${ }^{33}$, Weisskopf M. C. ${ }^{17}$, Zane S. ${ }^{18}$, Agudo I. ${ }^{19}$, Antonelli A. ${ }^{41}$, Attina P. ${ }^{1}$, Baldini L. ${ }^{2}$, Bykov A. ${ }^{20}$, Carpentiero R. ${ }^{60}$, Cavazzuti E. ${ }^{60}$, Churazov E. ${ }^{21}$, Del Monte E. ${ }^{1}$, De Martino D. ${ }^{22}$, Donnarumma I. ${ }^{1}$, Doroshenko V. ${ }^{14}$, Evangelista Y. ${ }^{1}$, Ferreira I. ${ }^{35}$, Gallo E. ${ }^{23}$, Grosso N. ${ }^{9}$, Kaaret P. ${ }^{24}$, Kuulkers E. ${ }^{35}$, Laranaga J. ${ }^{35}$, Latronico L. ${ }^{45}$, Lumb D. H. ${ }^{35}$, Macian J. ${ }^{16}$, Malzac J. ${ }^{25}$, Marin F. ${ }^{10}$, Massaro E. ${ }^{124}$, Minuti M. ${ }^{2}$, Mundell C. ${ }^{27}$, Ness J.U. ${ }^{91}$, Oosterbroek T. ${ }^{35}$, Paltani S. ${ }^{3}$, Pareschi G. ${ }^{15}$, Perna R. ${ }^{28}$, Petrucci P.-O. ${ }^{29}$, Pinazo H.

B. ${ }^{16}$, Pinchera M. ${ }^{2}$, Rodriguez J. P. ${ }^{16}$, Roncadelli M. ${ }^{30}$, Santovincenzo A. ${ }^{35}$, Sazonov S. ${ }^{31}$, Sgro C. ${ }^{2}$, Spiga D. ${ }^{15}$, Svoboda J. ${ }^{10}$, Theobald C. ${ }^{18}$, Theodorou T. ${ }^{18}$, Turolla R. ${ }^{32}$, Wilhelmi de Ona E. ${ }^{34}$, Winter B. ${ }^{18}$, Akbar A. M. ${ }^{36}$, Allan H. ${ }^{37}$, Aloisio R. ${ }^{38}$, Altamirano D. ${ }^{33}$, Amati L. ${ }^{39}$, Amato E. ${ }^{38}$, Angelakis E. ${ }^{40}$, Arezu J.42, Atteia J.-L. ${ }^{25}$, Axelsson M. ${ }^{43}$, Bachetti M.44, Ballo L. ${ }^{15}$, Balman S. ${ }^{46}$, Bandiera R. ${ }^{38}$, Barcons X.47, Basso S. ${ }^{15}$, Baykal A. ${ }^{46}$, Becker W.4 , Behar E. ${ }^{48}$, Beheshtipour B. ${ }^{49}$, Belmont R. ${ }^{25}$, Berger E. ${ }^{50}$, Bernardini F. ${ }^{51}$, Bianchi S. ${ }^{11}$,

Bisnovatyi-Kogan G. ${ }^{31}$, Blasi P. ${ }^{38}$, Blay P. ${ }^{16}$, Bodaghee A. ${ }^{52}$, Boer M. ${ }^{53}$, Boettcher M. ${ }^{54}$, Bogdanov S. ${ }^{55}$, Bombaci I. ${ }^{2}$, Bonino R. ${ }^{45}$, Braga J. ${ }^{56}$, Brandt W. ${ }^{57}$, Brez A. ${ }^{2}$, Bucciantini N. ${ }^{38}$ , Burderi L. ${ }^{58}$, Caiazzo I. ${ }^{59}$, Campana R. ${ }^{39}$, Campana S. ${ }^{15}$, Capitanio F. ${ }^{1}$, Cappi M. ${ }^{39}$, Cardillo M. ${ }^{1}$, Casella P. ${ }^{61}$, Catmabacak O. ${ }^{62}$, Cenko B. ${ }^{17}$, Cerda-Duran P. ${ }^{16}$, Cerruti C. ${ }^{16}$,

Chaty S. ${ }^{63}$, Chauvin M.43, Chen Y.64, Chenevez J. ${ }^{37}$, Chernyakova M. ${ }^{65}$, Cheung Teddy C.C. ${ }^{66}$, Christodoulou D. ${ }^{67}$, Connell P. ${ }^{16}$, Corbet R. ${ }^{68}$, Coti Zelati F. ${ }^{15}$, Covino S. ${ }^{15}$, Cui W. ${ }^{69}$ , Cusumano G. ${ }^{70}$, D'Ai A. ${ }^{71}$, D’Ammando F. ${ }^{39}$, Dadina M. ${ }^{39}$, Dai Z. ${ }^{64}$, De Rosa A. ${ }^{1}$, De Ruvo L. ${ }^{2}$, Degenaar N. ${ }^{33}$, Del Santo M. ${ }^{70}$, Del Zanna L. ${ }^{38}$, Dewangan G. ${ }^{72}$, Di Cosimo S. ${ }^{1}$, Di Lalla N. ${ }^{2}$, Di Persio G. ${ }^{1}$, Di Salvo T. ${ }^{71}$, Dias T. ${ }^{72}$, Done C. ${ }^{74}$, Dovciak M. ${ }^{10}$, Doyle G. ${ }^{26}$, Ducci L. ${ }^{3,14}$, Elsner R. ${ }^{17}$, Enoto T. ${ }^{75}$, Escada J. ${ }^{73}$, Esposito P. ${ }^{76}$, Eyles C. ${ }^{16}$, Fabiani S. ${ }^{1}$, Falanga M. ${ }^{77}$, Falocco S. ${ }^{78}$, Fan Y. ${ }^{79}$, Fender R. ${ }^{80}$, Feroci M. ${ }^{1}$, Ferrigno C. ${ }^{3}$, Forman W. ${ }^{50}$, Foschini L. ${ }^{15}$, Fragile C. ${ }^{81}$, Fuerst F. ${ }^{82}$, Fujita Y. ${ }^{83}$, Gasent-Blesa J. L. ${ }^{16}$, Gelfand J. ${ }^{51}$, Gendre B. ${ }^{84}$, Ghirlanda G. ${ }^{15}$, Ghisellini G. ${ }^{15}$, Giroletti M. ${ }^{39}$, Goetz D. ${ }^{63}$, Gogus E. ${ }^{62}$, Gomez J.-L. ${ }^{5}$, Gonzalez D. ${ }^{18}$, Gonzalez-Riestra R. ${ }^{85}$, Gotthelf E. ${ }^{55}$, Gou L. ${ }^{86}$, Grandi P. ${ }^{39}$, Grinberg V. ${ }^{87}$,

Grise F. ${ }^{9}$, Guidorzi C. ${ }^{15}$, Gurlebeck N. ${ }^{88}$, Guver T. ${ }^{89}$, Haggard D. ${ }^{90}$, Hardcastle M. ${ }^{92}$, Hartmann D. ${ }^{93}$, Haswell C. ${ }^{94}$, Heger A. ${ }^{95}$, Hernanz M. ${ }^{5}$, Heyl J. ${ }^{59}$, Ho L. ${ }^{95}$, Hoormann J.49, Horak J. ${ }^{10}$, Huovelin J. ${ }^{98}$, Huppenkothen D. ${ }^{51}$, Iaria R. ${ }^{71}$, Inam Sitki C. ${ }^{46}$, Ingram A. ${ }^{33}$, Israel G. ${ }^{61}$, Izzo L. ${ }^{99}$, Burgess M. ${ }^{43}$, Jackson M. ${ }^{100}$, Ji L. ${ }^{79}$, Ji L. ${ }^{102}$, Jiang J. ${ }^{101}$, Johannsen T. ${ }^{103}$, Jones C. ${ }^{50}$, Jorstad S. ${ }^{104}$, Kajava J. J. E. ${ }^{91}$, Kalamkar M. ${ }^{61}$, Kalemci E. ${ }^{62}$, Kallman T. ${ }^{75}$, Kamble A. ${ }^{50}$, Kislat F. ${ }^{49}$, Kiss M.43, Klochkov D. ${ }^{14}$, Koerding E. ${ }^{105}$, Kolehmainen M. ${ }^{74}$, Koljonen K. ${ }^{51}$, Komossa S. ${ }^{40}$, Kong A. ${ }^{106}$, Korpela S. ${ }^{98}$, Kowalinski M. ${ }^{8}$, Krawczynski H. ${ }^{49}$, Kreykenbohm I. ${ }^{107}$, Kuss M. ${ }^{2}$, Lai D. ${ }^{108}$, Lan M. ${ }^{64}$, Larsson J.43, Laycock S. ${ }^{67}$, Lazzati D. ${ }^{109}$, Leahy D. ${ }^{110}$, Li H. ${ }^{6}$, Li J. ${ }^{5}$, Li L.-X. ${ }^{111}$, Li T. ${ }^{6}$, Li Z. ${ }^{112}$, Linares M. ${ }^{87}$, Lister M. ${ }^{69}$, Liu H. ${ }^{113}$, Lodato G. ${ }^{114}$, Lohfink A. ${ }^{115}$, Longo F. ${ }^{116}$, Luna G. ${ }^{117}$, Lutovinov A. ${ }^{31}$, Mahmoodifar S. ${ }^{75}$, Maia J. ${ }^{73}$, Mainieri V. ${ }^{118}$, Maitra C. ${ }^{63}$, Maitra D. ${ }^{119}$, Majczyna A. ${ }^{120}$, Maldera S. ${ }^{45}$, Malyshev D. ${ }^{3}$, Manfreda A.45, Manousakis A. ${ }^{121}$, Manuel R. ${ }^{13}$, Margutti R. ${ }^{122}$, Marinucci A. ${ }^{11}$, Markoff S. ${ }^{33}$, Marscher A. ${ }^{123}$, Marshall H. ${ }^{87}$, Massaro F. ${ }^{45}$, McLaughlin M. ${ }^{125}$, Medina-Tanco G. ${ }^{126}$, Mehdipour M. ${ }^{127}$, Middleton M. ${ }^{115}$, Mignani R. ${ }^{76}$, Mimica P. ${ }^{16}$, Mineo T. ${ }^{70}$, Mingo B. ${ }^{128}$,

Space Telescopes and Instrumentation 2016: Ultraviolet to Gamma Ray, edited by Jan-Willem A. den Herder, Tadayuki Takahashi, Marshall Bautz, Proc. of SPIE Vol. 9905, 990515 (C) 2016 SPIE · CCC code: 0277-786X/16/\$18 - doi: 10.1117/12.2233046 
Miniutti G. ${ }^{13}$, Mirac S. M.46, Morlino G. ${ }^{129}$, Motlagh A. V. ${ }^{89}$, Motta S. E. ${ }^{80}$, Mushtukov A. ${ }^{33}$, Nagataki S. ${ }^{130}$, Nardini F. ${ }^{131}$, Nattila J. ${ }^{132}$, Navarro G. J. ${ }^{16}$, Negri B. ${ }^{60}$, Negro M.45, Nenonen S. ${ }^{133}$, Neustroev V. ${ }^{12}$, Nicastro F. ${ }^{61}$, Norton A. ${ }^{94}$, Nucita A. ${ }^{134}$, O'Brien P. ${ }^{128}$, O’Dell S. ${ }^{17}$, Odaka H. ${ }^{135}$, Olmi B. ${ }^{136}$, Omodei N. ${ }^{137}$, Orienti M. ${ }^{39}$, Orlandini M. ${ }^{39}$, Osborne J. ${ }^{128}$, Pacciani L. ${ }^{1}$, Paliya V. S. ${ }^{93}$, Papadakis I. ${ }^{139}$, Papitto A. ${ }^{61}$, Paragi Z. ${ }^{140}$, Pascal P. ${ }^{141}$, Paul B. ${ }^{142}$, Pavan L. ${ }^{3}$, Pellizzoni A.44, Perinati E. ${ }^{14}$, Pesce-Rollins M. ${ }^{2}$, Piconcelli E. ${ }^{61}$, Pili A. G. ${ }^{136}$, Pilia M.44, Pohl M. ${ }^{138}$, Ponti G. ${ }^{4}$, Porquet D. ${ }^{9}$, Possenti A. ${ }^{44}$, Postnov K. ${ }^{143}$, Prandoni I. ${ }^{61}$, Produit N. ${ }^{3}$, Puehlhofer G. ${ }^{14}$, Ramsey B. ${ }^{17}$, Razzano M. ${ }^{2}$, Rea N. ${ }^{5}$, Reig P. ${ }^{144}$, Reinsch K. ${ }^{145}$, Reiprich T. ${ }^{146}$, Reynolds M. ${ }^{24}$, Risaliti G. ${ }^{38}$, Roberts T. ${ }^{74}$, Rodriguez J. ${ }^{63}$, Rossi M. E. ${ }^{147}$, Rosswog S. ${ }^{148}$, Rozanska A. ${ }^{121}$, Rubini A. ${ }^{1}$, Rudak B. ${ }^{121}$, Russell D. ${ }^{51}$, Ryde F. ${ }^{43}$, Sabatini S. ${ }^{1}$, Sala G. ${ }^{149}$, Salvati M. ${ }^{38}$, Sasaki M. ${ }^{14}$, Savolainen T. ${ }^{150}$, Saxton R. ${ }^{91}$, Scaringi S.4 , Schawinski K. ${ }^{151}$ , Schulz N. S. ${ }^{87}$, Schwope A. ${ }^{152}$, Severgnini P. ${ }^{15}$, Sharon M. ${ }^{153}$, Shaw A. ${ }^{154}$, Shearer A. ${ }^{155}$, Shesheng X. ${ }^{124}$, Shih I.-C. ${ }^{156}$, Silva K. ${ }^{56}$, Silva R. ${ }^{73}$, Silver E. ${ }^{50}$, Smale A. ${ }^{75}$, Spada F. ${ }^{2}$, Spandre G. ${ }^{2}$, Stamerra A. ${ }^{157}$, Stappers B. ${ }^{158}$, Starrfield S. ${ }^{159}$, Stawarz L. ${ }^{160}$, Stergioulas N. ${ }^{161}$, Stevens A. ${ }^{33}$, Stiele H. ${ }^{97}$, Suleimanov V. ${ }^{14}$, Sunyaev R. ${ }^{21}$, Slowikowska A. ${ }^{162}$, Tamborra F. ${ }^{11}$, Tavecchio F. ${ }^{15}$, Taverna R. ${ }^{32}$, Tiengo A. ${ }^{163}$, Tolos L. ${ }^{5}$, Tombesi F. ${ }^{164}$, Tomsick J. ${ }^{165}$, Tong H. ${ }^{166}$, Torok G. ${ }^{167}$, Torres D. F. ${ }^{5}$, Tortosa A. ${ }^{11}$, Tramacere A. ${ }^{3}$, Trimble V. ${ }^{168}$, Trinchieri G. ${ }^{15}$, Tsygankov S. ${ }^{132}$, Tuerler M. ${ }^{3}$, Turriziani S. ${ }^{169}$, Ursini F. ${ }^{29}$, Uttley P. ${ }^{33}$, Varniere P. ${ }^{170}$, Vincent F. ${ }^{156}$, Vurgun E. ${ }^{89}$, Wang C. ${ }^{86}$, Wang Z. ${ }^{171}$, Watts A. ${ }^{33}$, Wheeler J. C. ${ }^{172}$, Wiersema K. ${ }^{128}$, Wijnands R. ${ }^{33}$, Wilms J. ${ }^{107}$, Wolter A. ${ }^{15}$, Wood K. ${ }^{66}$, Wu K. ${ }^{64}$, Wu X. ${ }^{18}$, Xiangyu W. ${ }^{64}$ , Xie F. ${ }^{43}$, Xu R. ${ }^{96}$, Yan S.-P. ${ }^{79}$, Yang J. ${ }^{67}$, Yu W. ${ }^{173}$, Yuan F. ${ }^{173}$, Zajczyk A.49, Zanetti D. ${ }^{2}$, Zanin R. ${ }^{174}$, Zanni C. ${ }^{157}$, Zappacosta L. ${ }^{61}$, Zdziarski A. A. ${ }^{121}$, Zech A. ${ }^{156}$, Zhang H. ${ }^{175}$, Zhang S. ${ }^{102}$, Zhang S. ${ }^{102}$, Zhang W. ${ }^{21}$, Zoghbi A. ${ }^{23}$

${ }^{1}$ IAPS/INAF, Via Fosso del Cavaliere 100, 00133 Rome, Italy

${ }^{2}$ University of Pisa \& INFN-Pisa, Largo B. Pontecorvo 3, 56127 Pisa, Italy

${ }^{3}$ Department of Astrononmy, University of Geneva, chemin d'Ecogia 16, 1290, Versoix, Switzerland

4MPI fuer extraterrestrische Physik, Giessenbachstrasse 1, D-85748, Garching, Germany

${ }^{5}$ Instituto de Astrofsica de Andaluca, CSIC, Apt. 3004, 18080 Granada, Spain

${ }^{6}$ Department of Engineering Physics and Center for Astrophysics, Tsinghua University, Beijing 100084, China

${ }^{7}$ Key Laboratory of Particle \& Radiation Imaging (Tsinghua University), Ministry of Education, China

${ }^{8}$ Space Research Centre, Polish Academy of Sciences Solar Physics Division, 51-622 Wroclaw, ul. Kopernika, Poland

${ }^{9}$ Observatoire Astronomique de Strasbourg, 11 rue de luniversite, 67000 Strasbourg, France

${ }^{10}$ Astronomical Institute Academy of Sciences of the Czech Republic, Bocni II 1401, CZ-14131

Prague, Czech Republic

${ }^{11}$ Dipartimento di Matematica e Fisica, Universita degli Studi Roma Tre, via della Vasca

Navale 84, 00146 Roma, Italy

${ }^{12}$ Tuorla Observatory, FI-21500 Piikkio, University of Turku, Finland

${ }^{13}$ National Institute of Aerospace Technology (INTA), Carretera de Ajalvir km. 4 - 28850

Torrejon de Ardoz, Spain;

14IAAT University of Tuebingen, Sand 1 - 72076 Tuebingen, Germany

${ }^{15}$ INAF-OA Brera, Via E. Bianchi 46, 23807 Merate (LC), Italy 
${ }^{16}$ University of Valencia, Av de Vicente Blasco Ibez, 13, 46010 Valencia, Spain

${ }^{17}$ NASA Marshall Space Flight Control Center, USA

${ }^{18}$ MSSL, Holmbury St Mary - RH5 6NT Dorking, Surrey, United Kingdom

${ }^{19}$ Instituto Astrofisica de Andalucia, Glorieta de la Astronoma (IAAC-CSIC), s/n., E-18008, Granada, Spain

${ }^{20}$ Ioffe Physico-Technical Institute, Politekhnicheskaya 26, 194021 St.Petersburg, Russia

${ }^{21}$ Max Planck Institute for Astrophysics, Garching, Karl-Schwarzschild-Str. 1, Postfach 1317, D-85741 Garching, Germany

${ }^{22}$ INAF-OA Capodimonte, Salita Moiariello, 1680131 Napoli, Italy

${ }^{23}$ University of Michigan Astronomy, 1085 S. University Ave, 305A West Hall, Ann Arbor MI 48109, United States

24Michigan state University, 567 Wilson Rd, MI 48824 East Lansing, United States

${ }^{25}$ IRAP, avenue du Colonel Roche, 9, BP 44346 Toulouse, France

${ }^{26}$ Armagh Observatory, College Hill, BT61 9DG Armagh, United Kingdom

${ }^{27}$ University of Bath, Claverton Down, Bath, BA2 7AY, United Kingdom

${ }^{28}$ Dept. of Physics and Astronomy, Stony Brook University, Stony Brook, NY 11794-3800, United States

${ }^{29}$ Institut de Planetologie et dAstrophysique de Grenoble, Universite Grenoble Alpes, CS 40700, 38058 Grenoble Cedex 9, France

${ }^{30}$ INFN Pavia, Via Agostino Bassi 6, 27100 Pavia, Italy

${ }^{31}$ Space Research Institute of the Russian Academy of Sciences, 84/32 Profsoyuznaya Str, Moscow, Russia

${ }^{32}$ Dept. of Physics and Astronomy, University of Padova, Via Marzolo 8, 35131 Padova, Italy

${ }^{33}$ Astronomical Institute Anton Pannekoek, University of Amsterdam, Science Park 904 - 1098 XH Amsterdam, The Netherlands

34Institut de ciences de l'espai, Campus UAB, Carrer de Can Magrans, S/N, 08193 Cerdanyola del Valles

${ }^{35}$ ESA/ESTEC, Keplerlaan 1, 2201 AZ Noordwijk, The Netherlands

${ }^{36}$ Effat University, P.O.BOX 34689, Jeddah 21478, Saudi Arabia

${ }^{37}$ National Space Institute, Technical University of Denmark, Elektrovej Bld 327, 2800 Kgs Lyngby, Denmark

${ }^{38}$ Arcetri Observatory, INAF, Largo Enrico Fermi 5, I-50125 Firenze, Italy

${ }^{39}$ INAF-IASF-Bologna, Via P. Gobetti 101, 40129 Bologna, Italy

${ }^{40}$ Max-Planck-Institut fr Radioastronomie, Auf dem Hgel 69, D-53121 Bonn (Endenich),

Postfach 20 24, D-53010 Bonn

${ }^{41}$ ASDC, Via del Politecnico snc, 00133 Rome, Italy

${ }^{42}$ Departament of Physics and Engineering Physics, Buein Zahra Technical University, Qazvin, Iran

${ }^{43} \mathrm{KTH}$ Royal Institute of Technology, Valhallavagen 79, 10044 Stockholm, Sweden

4 INAF-OA Cagliari, localita Poggio dei Pini, Strada 54, 09012 Capoterra, Italy

${ }^{45}$ INFN Torino, Via Pietro Giuria, 1, 10125 Torino, Italy

${ }_{46}$ Middle East Technical University, Ankara, Mah. Dumlupnar Blv. No:1 - 06800 ankaya

Ankara, Turkey

${ }_{47}$ Instituto de Fsica de Cantabria, E-39005 Santander, Spain

${ }^{48}$ Technion - Israel Institute of Technology, Technion City, Haifa 3200003, Israel 
${ }^{49}$ Washington University in St. Louis, 1 Brookings Dr, St. Louis, MO 63130, United States

${ }^{50}$ Harvard-Smithsonian Center for Astrophysics, 60 Garden St, Cambridge, MA 02138, United States

${ }^{51}$ NYUAD, PO Box 129188., Abu Dhabi, United Arab Emirates

${ }^{52}$ Georgia College, 231 W. Hancock St., Milledgeville, GA 31061, United States

${ }^{53}$ ARTEMIS UMR 7250, CNRS University of Nice Sophia-Antipolis, Observatoire de la Cote dAzur, CS 34229 F-06304 NICE, France

54 North-West University, Albert Luthuli \& University Drive, Mmabatho, Mahikeng, 2790,

South Africa

${ }^{55}$ Columbia University, 116th St \& Broadway, New York, NY 10027, United States

${ }^{56} \mathrm{INPE}$, Avenida dos Astronautas 1.758, Jd. da Granja, 12227-010 Sao Jose dos Campos, Brazil

${ }^{57}$ The Pennsylvania State University, University Park, Pennsylvania 16802, United States

${ }^{58}$ Cagliari University, Strada provinciale per Sestu KM 1, 09042 Monserrato, Italy

${ }^{59}$ Department of Physics and Astronomy, 6224 Agricultural Road, Vancouver, BC V6T 1Z1, Canada

${ }^{60}$ Agenzia Spaziale Italiana, Unita Osservazione dell'Universo, viale Liegi 2600198 Roma, Italy

${ }^{61}$ INAF-OA Roma, Via Frascati, 33 - 00040 Monte Porzio Catone, Italy

62Sabanci University, Orhanli-Tuzla 34956, Istanbul, Turkey

${ }^{63}$ CEA Saclay, DSM/IRFU/SAp, 91191 Gif sur Yvette, France

64Nanjing University, 22 Hankou Road Nanjing Jiangsu 210093, China

${ }^{65}$ School of Physical Sciences, Dublin City University, Glasnevin, Dublin 9, Ireland

${ }^{66}$ NRL, 4555 Overlook Ave. SW Washington, DC 20375-5352, United States

${ }^{67}$ University of Massachusetts Lowell, 1 University Ave, Lowell, MA 01852, United States

${ }^{68}$ University of Maryland, Baltimore County, 1000 Hilltop Circle, Baltimore, MD 21250,

United States

${ }^{69}$ Purdue University, 525 Northwestern Avenue, West Lafayette, IN 47907, United States

${ }^{70}$ INAF-IASF, Via Ugo La Malfa 153, 90146 Palermo, Italy

${ }^{71}$ Dipartimento di Fisica, Palermo University, Via Archirafi 36, 90123 Palermo, Italy

${ }^{72}$ Inter-University Centre for Astronomy and Astrophysics, Pune University Campus, Ganeshkhind, Pune, Maharashtra 411007, India

${ }^{73}$ LIP, Departamento de Fsica, Universidade de Coimbra, 3004-516 Coimbra, Portugal

${ }^{74}$ Durham University, Stockton Rd - DH1 3UP Durham, United Kingdom

${ }^{75}$ Goddard Space Flight Center, 8800 Greenbelt Rd., Md., 20771 Greenbelt, United States

${ }^{76}$ INAF-IASF-Milano, Via E. Bassini 15, I-20133 Milano, Italy

${ }^{77}$ ISSI, Hallerstrasse 6, 3012 Bern, Switzerland

${ }^{78}$ Dipartimento di Fisica, Universita degli Studi di Napoli Federico II, Via Cinthia, I-80126, Napoli, Italy

${ }^{79}$ Purple Mountain Observatory, CAS,2 West Beijing Road, Nanjing 210008, China

${ }^{80}$ Oxford University, Department of Physics, Clarendon Laboratory, Parks Road, Oxford, OX1 3PU, United Kingdom

${ }^{81}$ College of Charleston, 66 George St, Charleston, SC 29424, United States

${ }^{82}$ Caltech, MC 290-17, Pasadena, CA 91125, United States

${ }^{83}$ Osaka University, 1-1 Machikaneyama-cho, Toyonaka, Osaka, 560-0043, JAPAN

84 University of the Virgin Islands, College of Science, 2 John Brewers Bay, 00802 St Thomas 
VI, United States

${ }^{85}$ XMM-Newton Science Operation Centre, ESA, VILSPA, P.O.Box 50727, E-28080 Madrid, Spain

${ }^{86}$ National Astronomical Observatories, Chinese Academy of Sciences, 20A Datun Road, Chaoyang District, Beijing, China

${ }^{87}$ MIT, 77 Massachusetts Avenue, MA 02139 Cambridge, United States

88ZARM, Center of Applied Space Technology and Microgravity Universitat Bremen, Am Fallturm, 28359 Bremen, Germany

${ }^{89}$ Istanbul University, Beyazit, 34452 Fatih/stanbul, Turkey

${ }^{90}$ McGill University, 3550 University Street, Montreal, QC H3A 2A7, Canada

${ }^{91} \mathrm{ESA} / \mathrm{ESAC}$, Camino Bajo del Castillo, s/n., Urb. Villafranca del Castillo, 28692 Villanueva de la Canada, Madrid, Spain

${ }^{92}$ University of Hertfordshire, De Havilland Campus, Hatfield AL10 9EU, United Kingdom

${ }^{93}$ Clemson University, Clemson, SC 29634, United States

${ }^{94}$ Open University, Walton Hall, MK7 6AA Milton Keynes, United Kingdom

${ }^{95}$ Monash Centre for Astrophysics, School of Physics and School of Mathematical Sciences,

Monash University, Clayton VIC 3800, Australia

${ }^{96}$ Peking University, No.5 Yiheyuan Road Haidian District, 100871, Beijing, China

${ }^{97}$ Tsinghua University, 30 Shuangqing Rd, Haidian, Beijing, China

${ }^{98}$ Department of Physics, Division of Geophysics and Astronomy, P.O. Box 48, FI-00014

University of Helsinki, Finland

${ }^{99}$ Sapienza University and ICRA, p.le A. Moro 2, 00185, Rome, Italy

${ }^{100}$ Cardiff University, Cardiff CF10 3XQ, United Kingdom

${ }^{101}$ Fudan University, 220 Handan Rd, Yangpu, Shanghai, China

${ }^{102}$ Institute of High Energy Physics, 19B YuquanLu, Shijingshan district, Beijing, 1000049, China

${ }^{103}$ Perimeter Institute for Theoretical Physics, 31 Caroline Street North, Waterloo, ON, N2L 2Y5, Canada

104Institute for Astrophysical Research, Boston University, Address 725 Commonwealth Ave., Boston, MA 02215

${ }^{105}$ Radboud University Nijmegen, Comeniuslaan 4, 6525 HP Nijmegen, The Netherlands

${ }^{106}$ National Tsing Hua University, No. 101, Section 2, Guangfu Rd, East District, Hsinchu City, Taiwan

${ }^{107}$ University of Erlangen-Nuremberg, Schlossplatz 4, 91054 Erlangen, Germany

${ }^{108}$ Cornell University, Space Building, NY 14853 Ithaca, United States

${ }^{109}$ Department of Physics, Oregon State University, 301 Weniger Hall, Corvallis, OR 97331, United States

${ }^{110}$ University of Calgary, 2500 University Dr NW, Calgary, AB T2N 1N4, Canada

${ }^{111}$ Kavli Institute for Astronomy and Astrophysics, Qinghua W Rd, Haidian, Beijing, China

${ }^{112}$ Department of Physics, Xiangtan University, china

${ }^{113}$ Guangxi University, Guangxi, Nanning, Xixiangtang, China

${ }^{114}$ Dipartimento di Fisica, Universita degli Studi di Milano, Via Celoria 16, 20133 Milano, Italy

${ }^{115}$ Institute of Astronomy, University of Cambridge, Madingley Road, Cambridge CB3 0HA,

United Kingdom

${ }^{116}$ University of Trieste, Via Alfonso Valerio, 32 - 34128 Trieste, Italy 
${ }^{117}$ IAFE/CORNICET, Godoy Cruz 2290, CABA, Argentina

${ }^{118}$ ESO, Karl-Schwarzschild-Strasse 2, D-85748 Garching bei Munchen, Germany

${ }^{119}$ Dept. of Physics and Astronomy, Wheaton College, 501 College Ave. Wheaton, IL 60187, United States

${ }^{120}$ National Centre for Nuclear Research, Andrzeja Soltana 7, 05-400 Otwock-Swierk, Poland

${ }^{121}$ Copernicus Astronomical Center, Bartycka 18, Warsaw, Poland

${ }^{122}$ New York University, New York, NY, United States

${ }^{123}$ Boston University, Boston, MA 02215, United States

124 Universita degli Studi di Roma La Sapienza, Piazzale Aldo Moro 5, 00185, Roma, Italy

${ }^{125}$ West Virginia University, Morgantown, WV 26506, United States

${ }^{126}$ Instituto de Ciencias Nucleares, UNAM, Apartado Postal 70-543, Ciudad Universitaria, Mexico D.F. 04510, Mexico

${ }^{12}{ }^{2}$ SRON, Sorbonnelaan 2, 3584 CA Utrecht, The Netherlands

${ }^{128}$ Leicester University, University Road, LE1 7RH Leicester, United Kingdom

${ }^{129}$ Gran Sasso Science Institute, Viale Francesco Crispi, 7, L'Aquila, Italy

${ }^{130}$ RIKEN, Japan

${ }^{131}$ Keele University, Keele ST5 5BG, United Kingdom

132Tuorla Observatory, Vaisalantie 20, 21500 Piikkio, Finland

${ }^{133}$ Oxford Instruments Analytical Oy, Tarvonsalmenkatu 17, Espoo, Finland

${ }_{134}$ Lecce University, Piazza Tancredi n7, 73100, Lecce, Italy

${ }^{135}$ ISAS/JAXA, 3-1-1 Yoshinodai, Chuo-ku, Sagamihara, Kanagawa 252-5210, Japan

${ }^{136}$ University of Florence, Piazza di San Marco, 4, 50121 Firenze, Italy

${ }^{137}$ Kipac, 2575 Sand Hill Road, M/S 29, Menlo Park, CA 94025, United States

${ }^{138}$ DPNC, Geneve University, Quai Ernest-Ansermet 30, 1205 Geneva, Switzerland

${ }^{139}$ Physics Department, University of Crete, GR-710 03 Heraklion, Greece

${ }^{140}$ Joint Institute for VLBI in Europe (JIVE), Postbus 2, 7990 AA Dwingeloo, The

Netherlands

${ }^{141}$ Observatoire Midi-Pyrenees, 14 Avenue Edouard Belin, 31400 Toulouse, France

142Raman Research Institute, C. V. Raman Avenue, 560080 Sadashivanagar, India

${ }^{14}{ }^{3}$ Sternberg Astronomical Institute, Moscow M.V. Lomonosov State University, 119992

Moscow, Russia

144Foundation for Research \& Technology Hellas, Leof. Plastira 100, Iraklio 700 13, Greece

${ }^{145}$ Georg-August-Universitaet Goettingen, Institut fuer Astrophysik, Friedrich-Hund-Platz 1, 37077 Gottingen, Germany

${ }^{146}$ Argelander-Institut fuer Astronomie, Auf dem Hugel 71, 53121 Bonn, Germany

${ }^{147}$ Leiden Observatory, Niels Bohrweg 2, NL-2333 CA Leiden, The Netherlands

${ }^{148}$ Stockholm University, Universitetsvagen 10, 11418 Stockholm, Sweden

${ }^{149}$ Polytechnic University of Catalonia, Campus Nord, Calle Jordi Girona, 1-3, 08034

Barcelona, Spain

${ }^{150}$ Aalto University, Metsahovi Radio Observatory, P.O.Box 13000, FI-00076, Aalto, Finland

${ }^{151}$ ETH Zurich, Wolfgang-Pauli-Str. 27, 8093 Zurich, Switzerland

${ }^{152}$ Leibniz-Institut fuer Astrophysik Potsdam, An der Sternwarte 16, D-14482 Potsdam,

Germany

${ }^{153}$ University of Alberta, Edmonton, Alberta, T6G 2E9, Canada

154 University of Southampton, Southampton, SO17 1BJ, United Kingdom 
${ }^{155}$ National University of Ireland Galway, University Road, Galway, Ireland

${ }^{156}$ Paris Observatory, 61 Avenue de l'Observatoire, 75014 Paris, France

${ }^{157}$ INAF-OA Torino, Corso Savona, 10024 Moncalieri, Torino, Italy

${ }^{158}$ University of Manchester, Booth Street West, M15 6PB Manchester, United Kingdom

${ }^{159}$ School of Earth and Space Exploration, P. O. Box 871404, Arizona State University Tempe,

Arizona 85287-1404, United States

${ }^{160}$ Astronomical Observatory of the Jagiellonian University, Fort 38 Skala, Orla 171, 30-244

Krakow, Poland

${ }^{161}$ Aristotle University of Thessaloniki, Thessaloniki 541 24, Greece

162 Janusz Gil Institute of Astronomy, ul. Szafrana 2, 65-516 Zielona Gora, Poland

${ }^{163}$ IUSS, Istituto Universitario di Studi Superiori di Pavia, Palazzo del Broletto, Piazza della

Vittoria n.15, 27100 Pavia, Italy

164 University of Maryland, Department of Astronomy, College Park, MD 20742-2421, United

States

${ }^{165}$ University of California, Berkeley, Space Sciences Laboratory, 7 Gauss Way, Berkeley, CA 94720-7450

${ }^{166}$ Xinjiang Astronomical Observatory, Chinese Academy of Sciences, 150 Science 1-Street,

Urumqi, Xinjiang 830011, China

${ }^{16}{ }^{7}$ Silesian University in Opava, Na Rybnicku 626/1, 74601 Opava, Czech Republic

${ }^{168}$ University of California Irvine, Physics-Astronomy Department, 4575 Physics Irvine CA 92697-4575, California, United States

${ }^{169}$ University of Rome Tor Vergata, Via della Ricerca Scientifica 1, I-00133 Roma, Italy

${ }^{170}$ APC, AstroParticule \& Cosmologie, UMR 7164 CNRS/N2P3, Universit Paris Diderot, 75205 Paris Cedex 13, France

${ }^{171}$ Tongji University, Shanghai 200092, China

${ }^{172}$ The University of Texas at Austin, Department of Astronomy, 2515 Speedway, Stop C1400,

Austin, Texas 78712-1205, United States

${ }^{173}$ Shanghai Astronomical Observatory, 80 Nandan Road, Shanghai 200030, China

${ }^{17}$ Institut fuer Kernphysik, Technische Universitat Darmstadt, 64289 Darmstadt, Germany

${ }^{175}$ Theoretical Division, Los Alamos National Laboratory, Los Alamos, NM 87545, United

States

\begin{abstract}
XIPE, the X-ray Imaging Polarimetry Explorer, is a mission dedicated to X-ray Astronomy. At the time of writing XIPE is in a competitive phase A as fourth medium size mission of ESA (M4). It promises to reopen the polarimetry window in high energy Astrophysics after more than 4 decades thanks to a detector that efficiently exploits the photoelectric effect and to X-ray optics with large effective area. XIPE uniqueness is time-spectrallyspatially-resolved X-ray polarimetry as a breakthrough in high energy astrophysics and fundamental physics. Indeed the payload consists of three Gas Pixel Detectors at the focus of three X-ray optics with a total effective area larger than one XMM mirror but with a low weight. The payload is compatible with the fairing of the Vega launcher. XIPE is designed as an observatory for X-ray astronomers with $75 \%$ of the time dedicated to a Guest Observer competitive program and it is organized as a consortium across Europe with main contributions from Italy, Germany, Spain, United Kingdom, Poland, Sweden.
\end{abstract}

Keywords: X-ray Astronomy, Polarimetry, X-ray optics, Gas Pixel Detector 


\section{INTRODUCTION}

$\mathrm{X}$-ray polarimetry promises to provide a wealth of information. Indeed emission processes in this energy band are expected to provide polarized radiation. However, even if the radiation is emitted thermally, and therefore intrinsically not polarized, the scattering in a-spherical plasma which are common in X-ray Astronomy provide polarized radiation. Also X-ray polarimetry probes environment with magnetic fields much larger than that obtainable on the Earth allowing for detecting phenomena of birefringence not yet discovered in ground experiments. Here below we provide some early bibliography on these topics.

1. Emission processes themselves: cyclotron, synchrotron, non-thermal bremsstrahlung ${ }^{1-3}$

2. Scattering on aspherical accreting plasmas: disks, blobs, columns ${ }^{3-5}$

3. Vacuum polarization and birefringence through extreme magnetic fields ${ }^{6-8}$

Polarimetry would allow for determining the geometry of sources on angular scales not accessible with current or planned X-ray astronomy imaging missions. A positive measurement will remove geometrical degeneracies in $\mathrm{X}$-ray binaries, in isolated pulsar and magnetars. Indeed, nowadays, geometry is still a parameter in a fit of a light-curve or of a spectrum and it is not the result of a direct measurement.

\section{MODULATION CURVE AND MINIMUM DETECTABLE POLARIZATION}

An X-ray polarimeter measures a modulation curve, that is the response of a polarization sensitive device to an angle related to the polarization angle of the incoming beam. We fit the modulation curve with a $\cos ^{2}$ function. We then normalize the modulation amplitude using the modulation factor, which is the modulation amplitude for $100 \%$ polarized beam, deriving the polarization. From the position of the peaks of the modulation curve we derive the polarization angle. Another important parameter is the Minimum Detectable Polarization (MDP) which is the level over which we can reject the hypothesis of absence of polarization at $99 \%$ confidence level and expressed as in equation 1:

$$
M D P(99 \%)=\frac{4.29}{\mu S} \sqrt{\frac{S+B}{T}}
$$

where $\mathrm{B}$ is the background counting rate (negligible for focal plane experiments as in the case of a GPD at the focus of an X-ray optics), $\mathrm{T}$ is the observing time, $\mathrm{S}$ is the source counting rate and $\mu$ is the modulation factor. ${ }^{9}$

As can be seen in table 1 the total number of counts for reaching an MDP of $1 \%$ is three order of magnitude larger than that needed to detect a source or to measure a spectral slope.

Table 1. Different number of counts for different kind of measurements. We assumed $\mu$ is 0.5 for the $1 \%$ MDP

\begin{tabular}{|l|l|}
\hline Source detection & 10 counts \\
\hline Spectral slope & 100 counts \\
\hline $1 \%$ MDP & counts $>700000$ \\
\hline
\end{tabular}

Therefore X-ray polarimetry deserves a dedicated mission with detectors developed at this aim. Each time polarimetry is tempted as a byproduct of instruments not specifically designed for this, at best, low significance measurements, (if not in contradiction), are produced. So far, the only positive measurement available is indeed the one obtained with the polarimeter on-board OSO-8 based on Bragg diffraction by the group of Robert Novick. ${ }^{10}$ We recall that the measurement of the X-ray polarization of the Crab Nebula has a 19- $\sigma$ significance at $2.6 \mathrm{keV}$ and $10-\sigma$ significance at $5.2 \mathrm{keV}$. Only very coarse upper limits ${ }^{11}$ were measured by OSO-8 (except that on Sco X-12). These upper limits are not particularly useful. 


\section{MODERN X-RAY POLARIMETRY}

The modern X-ray polarimeters exploit efficiently the photoelectric effect in gas. The photoelectron from a Kshell is ejected preferentially along the direction of the polarization vector of the incoming beam. If the track is finely resolved and efficiently imaged the emission direction can be reconstructed by applying simple algorithms. We, at this aim, devised and built the Gas Pixel Detector (GPD) to be sensitive in 1-10 keV energy band. In this energy band, basically, all the scientific cases for X-ray polarimetry can be eventually investigated (see table 2), and also conventional X-ray optics are efficient and sources are sufficiently bright.

Polarimeters based on multi-layer mirrors are efficient below $1 \mathrm{keV}$ and, while they may be very sensitive for blazars and isolated pulsars, this energy range suffers of interstellar absorption. Moreover this is not the proper energy band for studying effects of General Relativity in strong gravity regime or phenomena characterized by Compton reflection. The energy band above $10 \mathrm{keV}$ is, instead, particularly suited for studying reflection phenomena and for polarimetry of cyclotron lines in X-ray pulsator. However it does not allow for probing magnetars, including the QED effects of birefringence, or Black Hole X-ray Binaries and general relativistic effects in the strong gravity regime.

Table 2. Scientific goals for X-ray polarimetry. Cyclotron lines are observed above $10 \mathrm{keV}$ except, possibly in one

\begin{tabular}{|c|c|c|c|c|}
\hline Scientific goals & Sources & $<1 \mathrm{keV}$ & $1-10$ & $>10 \mathrm{keV}$ \\
\hline \multirow{4}{*}{$\begin{array}{l}\text { Acceleration } \\
\text { phenomena }\end{array}$} & PWN & yes (but absorp.) & yes & yes \\
\hline & SNR & no & yes & yes \\
\hline & jet $(\mu \mathrm{QSO})$ & yes (but absorp.) & yes & yes \\
\hline & jet (Blazars) & yes & yes & yes \\
\hline \multirow{4}{*}{$\begin{array}{l}\text { Emission } \\
\text { in strong } \\
\text { magnetic } \\
\text { fields }\end{array}$} & WD & difficult & yes & yes (difficult) \\
\hline & AMS & no & yes & yes \\
\hline & X-ray pulsator & difficult (absorp.) & yes (no cyclotron?) & yes \\
\hline & Magnetar & yes (better) & yes & no \\
\hline \multirow{2}{*}{$\begin{array}{l}\text { Scattering } \\
\text { in a- } \\
\text { spherical } \\
\text { geometries }\end{array}$} & Corona in XRB \& AGNs & yes (but absorp.) & yes & difficult \\
\hline & $\mathrm{X}$-ray reflection nebulae & no & yes (long exposure) & yes \\
\hline \multirow{4}{*}{$\begin{array}{l}\text { Fundamental } \\
\text { Physics }\end{array}$} & QED (magnetar) & yes(better) & yes & no \\
\hline & $\mathrm{GR}(\mathrm{BH})$ & no & yes & no \\
\hline & QG (Blazars) & difficult & yes & yes \\
\hline & Axions (Blazars, Clusters) & yes? & yes & difficult \\
\hline
\end{tabular}

\section{THE DETECTOR CAPABILITY}

The detector is designed ${ }^{13-15}$ as a sealed gas cell. A $50 \mu \mathrm{m}$ thick beryllium window is glued on a titanium frame and the latter onto a MACOR spacer. Underneath, the Gas Electron Multiplier and its supporting frame are glued on the KIOCERA package. An ASIC-CMOS, developed at this aim, is internally bonded to the package. The top layer of the multi-layer ASIC is an hexagonally patterned (105400 pixels) collection plane. Below each pixel there is a complete analogue chain. A set of local triggers define the region of interest that surrounds the track. Only a fraction of pixels (600-800) are serially readout reducing dramatically the dead time fraction. Three generation of ASICs were produced. The current ASIC is the first self triggered one with its trigger output signal allowing for time resolved polarimetry. Modulation factor and energy resolution were measured to be basically constant for at least three years of operation so this design is particularly suited for a long term space mission. The modulation factor is found in agreement with the Monte Carlo expectations and a cut on 20 $\%$ of data allows for the best sensitivity. The energy resolution is found compliant with the requirement of $20 \%$ (point source) and $25 \%$ extended sources. Some experimental results are shown in figure 1 while details on the performances can be found in this proceedings. ${ }^{16}$ 

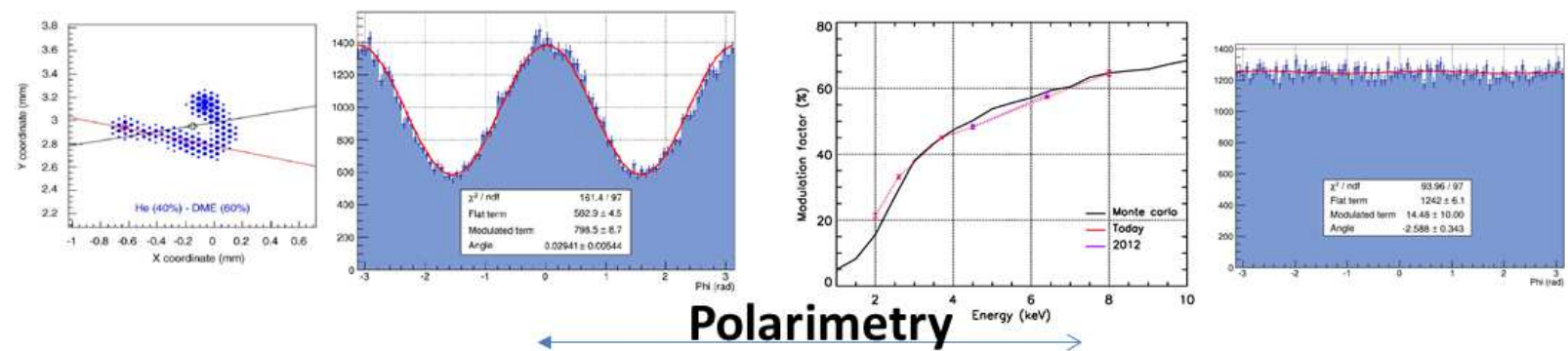

Polarimetry
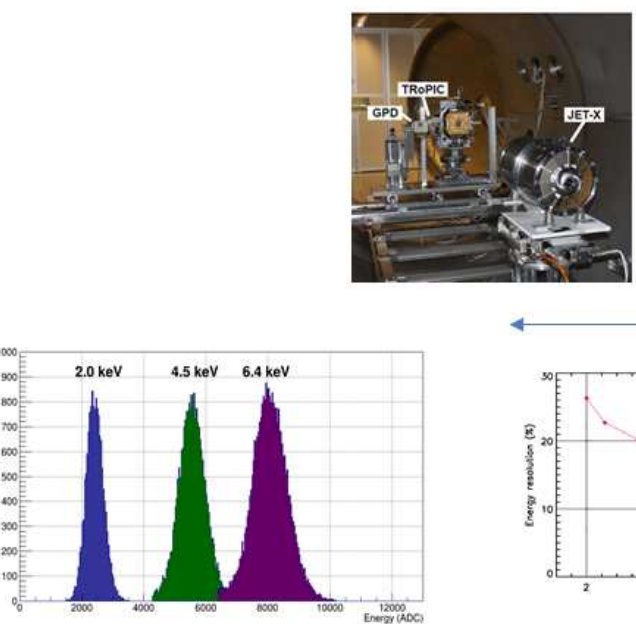

Imaging
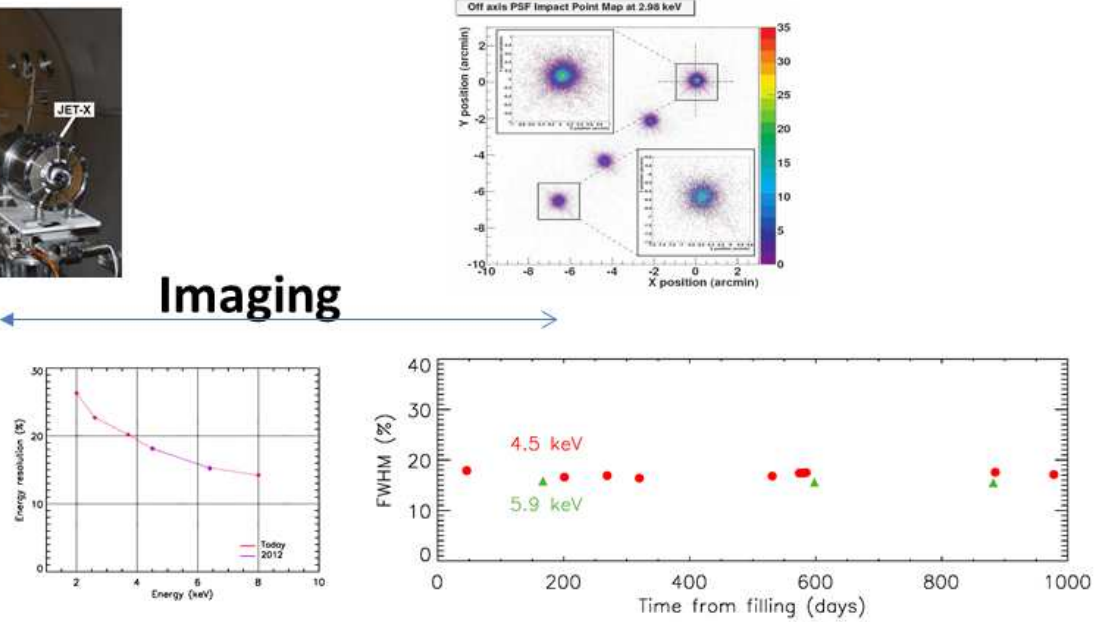

Spectroscopy

Figure 1. (Top) From left: an image of a photoelectron track; a modulation curve at 3.7 keV; the modulation factor as a function of energy; a modulation curve for an unpolarized ${ }^{55} \mathrm{Fe}$ source. Middle. From left: the GPD at the focus of a JET-X optics together with the CCD and the ROSAT PSPC; the on-axis and off-axis images. Bottom From left: the spectra of sources at 2.0, 4.5 and $6.4 \mathrm{keV}$; the behavior of the energy resolution with energy; the behavior of the energy resolution with time.

The imaging capabilities of the detector were measured either in laboratory with a narrow pencil beam ${ }^{17}$ and at the PANTER X-ray test facility. ${ }^{18}$ We disentangled the various components concurring to the overall angular resolution confirming the expectation ${ }^{19}$ that the main contribution is provided by the performances of the optics. The second contribution for importance is given by the inclined penetration of the photons in the 1-cm thick drift region, due to the narrow incidence optics, while the intrinsic position resolution of the detector provides a negligible contribution. We note here that the GPD met the strict requirements for operating in high vacuum and in cleanness environment of the PANTER test facility.

\section{THE PATH OF XIPE TO THE $4^{T H}$ MEDIUM SIZE CALL}

Thanks to the invention of the GPD, many missions were proposed answering to call of different international space agencies like ASI, CNSA, NASA and ESA. POLARIX, ${ }^{20}$ a missions similar to XIPE but with X-ray optics with much smaller effective area successfully accomplished phase A and it was selected to be one of the two small missions of ASI. Unfortunately the program was then cancelled.

A polarimeter based on GPD technology was foreseen for XEUS/IXO but then Athena, selected as the second large ESA mission, has not a polarimeter on-board. GEMS ${ }^{21,22}$, which uses a technique ${ }^{23}$ that privileges the quantum efficiency with respect to imaging, was selected for a flight but then, in 2012, it was discontinued for programmatic reasons. In 2012, after the discontinuation of GEMS, we decided to answer to a call for the $1^{s t}$ small mission of ESA (S1) proposing XIPE ${ }^{17}$ (the X-ray Imaging Polarimetry Explorer) a mission consisting of 
a payload with two GPDs and two already available JET-X optics. In addition we proposed two GPDs, tuned at higher energies for polarimetry of solar flares, and one X-ray photometer. XIPE was short-listed, it received a good scientific raking, but judged much too costly.

In 2014 ESA issued an Announcement of Opportunity for the 4th Scientific Mission of Medium Size (M4) with a budget of 450 Meuros (plus national contributions). At variance with precedent medium size calls, the budget is reduced by about $20 \%$ reflecting a faster track to launch and no further competition for the instruments. This call requires therefore, a high readiness for the payload. We proposed a larger version of XIPE compared with $\mathrm{S} 1$ proposal, increasing either the number of optics, from two to three, and their effective area exploiting the thin shell technologies. XIPE name was maintained. Therefore XIPE for M4 is much more sensitive than XIPE proposed to S1.

At the present time three missions have been selected on 2015 for phase A study:

- XIPE: an X-ray Imaging Polarimeter based on GPD technology.

- ARIEL: a mission for the spectroscopy of Exoplanets.

- THOR: a mission to study turbulence in the Solar Wind.

In the second quarter of 2017 one of these three missions will be selected for the phase B1, and, provided a successful adoption lasting one year, it will fly in 2026. In the following we will describe XIPE the X-ray Imaging Polarimetry Explorer currently in phase A for M4.

\section{XIPE MISSION}

$\mathrm{XIPE}$ is a mission which peculiarity is to perform time-spatially-spectrally resolved X-ray polarimetry as a break-through in high energy astrophysics and fundamental physics. It takes advantage of precedent phase A for POLARIX and XEUS/IXO. XIPE is more similar to POLARIX in terms of configuration but with three new optics made with the technology of replication of nickel electroformed thin-shells. The instrument is composed of three Detector Units (DUs) and three Back End Electronics Units (BEEUs) and of one Instrument Control Unit (ICU). Each DU consists of one GPD surmounted by a Filter Wheel (FW) which hosts the calibration system. In the following we describe the XIPE payload items.

\subsection{X-ray optics}

Each of the three XIPE Mirror Units (MUs) comprises an optics with 30 mirror shells with diameters from 407 $\mathrm{mm}$ to $181 \mathrm{~mm}$. They features a double-cone approximation of the Wolter-I profile with $300 \mathrm{~mm}$ of parabolic segment plus $300 \mathrm{~mm}$ of hyperbolic segment. The focal length is $4.0 \mathrm{~m}$ and it is chosen so that XIPE can fit in the fairing of the Vega launcher. This is an improvement in terms of effective area especially at higher energies with respect to the optics proposed at the M4 call. The precedent baseline had a Mirror Unit of 27 shells and 3.5 meter focal length each. These optics have a ratio between the thickness and the diameter of the shell a factor of 5 smaller than that of JET-X (the optics foreseen for POLARIX) providing a much more favorable performance to mass ratio. The characteristics of the mirror are shown in table 3 .

The design has been performed assuming a thickness/radius ratio of $1.7 \times 10^{-3}, 10$ arcmin diameter unobstructed Field of View (FoV), 10\% structure vignetting. An Half Energy Width (HEW) of 20 arcsec is assumed based on NHXM and eRosita mirror performances and on their integration procedure. ${ }^{24}$ The integration procedure allows the assembled optics for basically maintaining the same HEW of the single shells. At this regard we note that the mirrors of eRosita have a better HEW (16 arcsec on-axis $\left.{ }^{25}\right)$, thus we consider the assumed HEW of the XIPE mirrors is conservative. 
Table 3. Characteristics of the X-ray optics of XIPE

\begin{tabular}{|l|l|}
\hline Geometrical profile & Wolter I \\
\hline Focal length & $4 \mathrm{~m}$ \\
\hline Mirror length & $60 \mathrm{~cm}$ \\
\hline Max/Min shell diam. & $407-180 \mathrm{~mm}$ \\
\hline no. of spiders & 2 \\
\hline no. of spoke of spiders & 20 \\
\hline Spider and case materials & Steel \\
\hline Wall materials & Electroformed NiCo \\
\hline Coating & Ir $(30 \mathrm{~nm})+\mathrm{C}(10 \mathrm{~nm})$ bilayer \\
\hline Weight $(1 \mathrm{MM})$ & $68 \mathrm{~kg}$ \\
\hline Power & $50 \mathrm{~W}$ \\
\hline
\end{tabular}

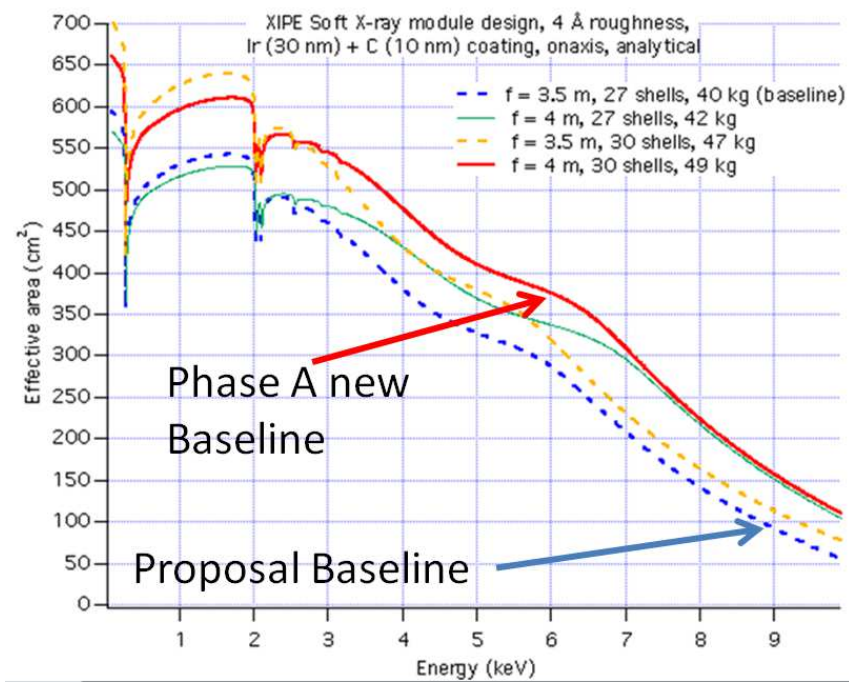

Figure 2. The effective area of one mirror module. The blue curve is the effective area adopted in the proposal. The red curve is the one now adopted in phase A. This new baseline has a focal length of $4 \mathrm{~m}$ and 30 shells.

\subsection{The Detector Unit and the Back End Electronics}

The Focal plane comprises three DUs coupled to their BEEU. Each DU includes the GPD and the calibration system (see figure 3) in their housing. The GPD is filled with a mixture of He-DME (20\%-80\%) at 1-bar with $1 \mathrm{~cm}$ of drift length. A Peltier cell keeps the temperature of the GPD within the requirement of stability of $1^{\circ} \mathrm{C}$. The $\mathrm{FW}$ on top of it, is equipped with a set of calibration sources, diaphragms and filters for a total of 8 positions selectable via telecommand during observation. One of this source is a polarized one exploiting Bragg diffraction at $45^{\circ}$ either at $2.6 \mathrm{keV}$ and at $4.5 \mathrm{keV}$. The other calibration sources allow for calibrating the gain and as a check of absence of spurious polarization. A closed position allows for gathering the internal background.

The BEEU must be close to the GPD by less than $20 \mathrm{~cm}$. A laboratory BEEU is routinely used for on-ground calibration purposes and some of its components, such as the FPGA and the ADC, will be changed, for the flight design, to space qualified ones.

Here below in a synthetic way we describe the functionality of the BEEU: 
- Manage the ASIC power supply and readout.

- Provide the High Voltage to the GEM.

- Interface the Peltier Driver for the detector temperature control.

- Analogue to Digital convert the data from the ASIC.

- Analogue to Digital convert the housekeeping data.

- Zero-suppress the empty pixels for each event.

- Time tag the event with $1 \mathrm{MHz}$ clock and the PPS signal.

- Format the data and send it to the Instrument Control Unit.

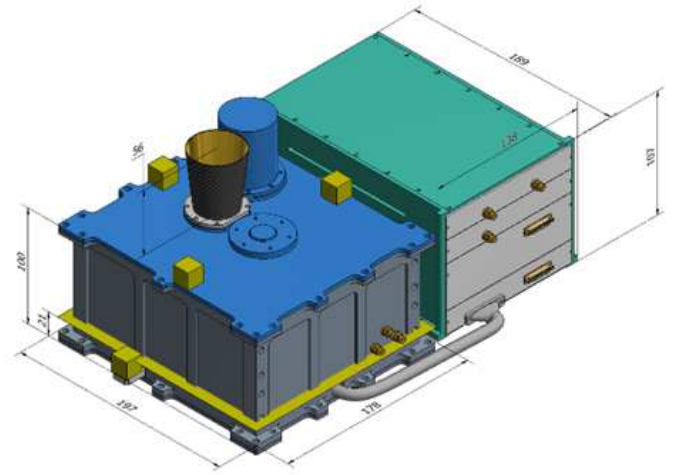

(a)

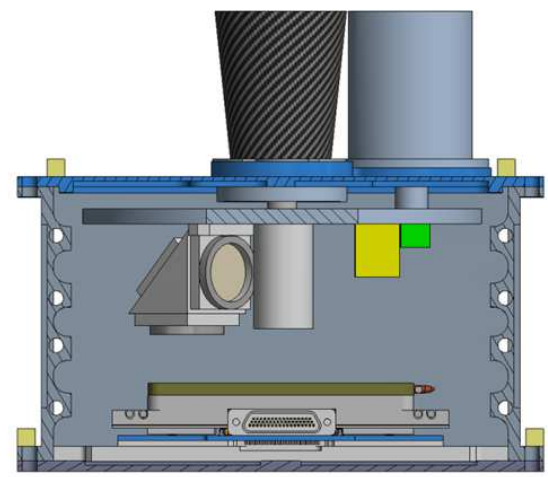

(b)

Figure 3. (a). The Detector Unit which comprises the Gas Pixel detector and the Filter Wheel. On its side the Back End Electronics Unit(b). The exploded view of the Detector Unit with on top the Filter Wheel equipped with a set of calibration sources including the polarized X-ray source and on the bottom the Gas Pixel Detector.

\subsection{The Focal Plane Assembly Support Structure}

The detector units and BEEU are arranged in a $120^{\circ}$ symmetry supported by an instrument platform at a distance with respect to the Service Module (SVM) and connected to it by means of a Structural Tube (ST). The Focal Plane Assembly Structure (FSS, baseline configuration see figure 4) consists of two different stacked panels. Each panel consists of Carbon Fiber skins and a cardboard honeycomb. This solution accommodates the DU and the BEEU that have different thermal requirements. Indeed the GPD has an operative working temperature of $5-25{ }^{\circ} \mathrm{C}$ and a stability requirement of $1{ }^{\circ} \mathrm{C}$ while the BEEU has a wider operative/non operative temperature range and a much less stringent stability requirement. This configuration solves this issue because the DU and the BEEU are thermally decoupled. Two titanium rings keep this structure tight, and allow for interfacing the FSS with the star tracker and the ST.

While writing this proceeding, a trade-off study has being performed. One aspect of this trade-off is the compliance with the following requirement: (1) capability of alignment of the GPD, with respect to the optics, (2) capability of replacing one DU if needed and (3) capability to replace/insert the calibration sources prior integration in the fairing. These requirements are suggesting a different solution, similar to the one adopted to eRosita, with respect to the baseline described above. This solution foresee of connecting the DU from the external side of the FSS with suitable cuts and reinforcement on it. 


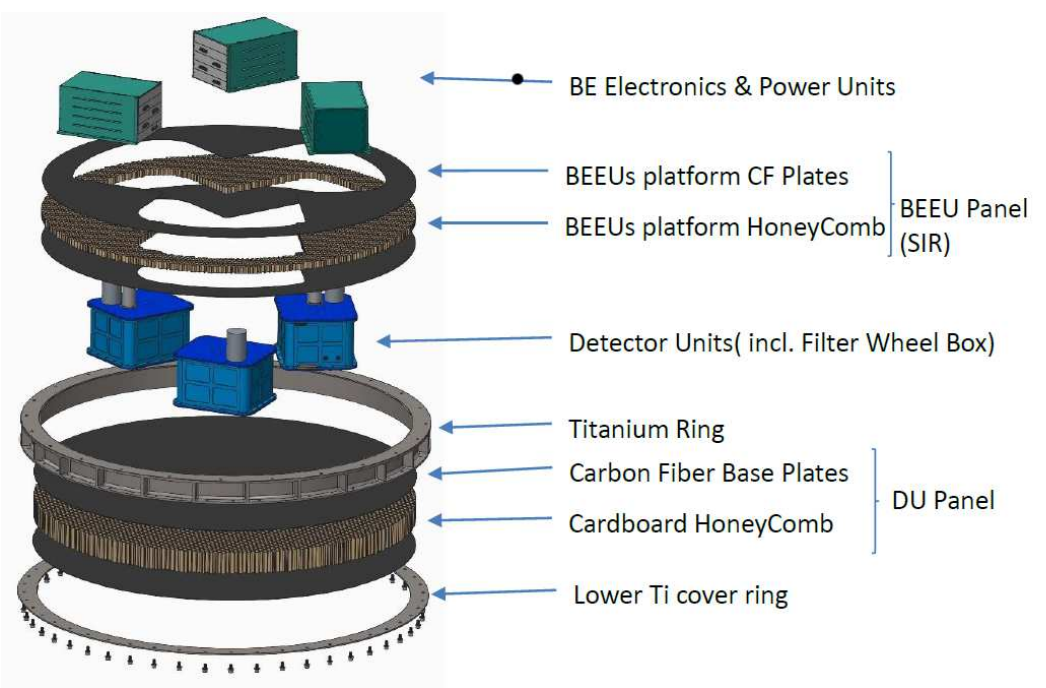

Figure 4. The Focal Plane Assembly Structure. This design represents the XIPE baseline while a trade-off study is underway.
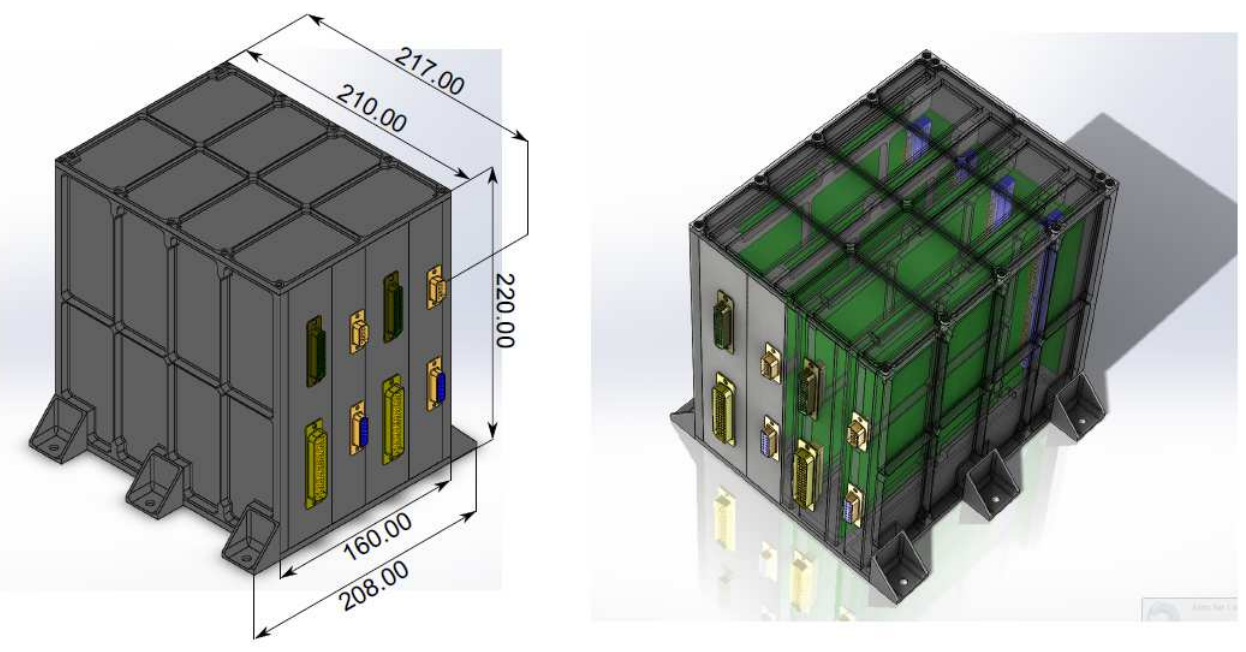

Figure 5. The Instrument Control Units. Double Eurocard standard are foreseen.

\subsection{Instrument Control Unit}

The ICU is a data processing box which also controls the three BEEUs. It consists of a Micro-processor \& Memory board, a power distribution boards, a Filter Wheel driver board and a Peltier driver Board (see fig. 5).

The ICU is in charge of:

- managing the individual SpaceWire interfaces to the three BEEUs.

- Manage the Pulse-per-Second (PPS) signal and the $1 \mathrm{MHz}$ clock for the on-board time and synchronization of the BEEU.

- Retrieve science data and housekeeping (HK) data from the BEEUs.

- Store data into the Mass Memory (32 GB) before sending them to on-board data handling for downlink. 
- Generate the Quick Look Analysis (QLA) data from the science data.

- Take care of TM/TC Spacewire interface to the spacecraft, of the execution and of the distribution of commands.

- Manage the payload operative modes.

- Perform instrument health monitoring.

- drive the Peltier cooler and the FW.

Moreover the ICU will:

- Receive the non-regulated primary power from the Service Module.

- Generate and distribute the regulated low voltages to the BEEs.

- Manage the high voltage of the GPD basing on the rate of an on-board radiation monitor during passages through the SAA or for a solar flare

- Operate the filter wheels (FWs).

- Operate the Peltier coolers

- Generate HK data about voltage, current, temperature and operational parameters.

QLA data are data processed on-board consisting of spectra, light-curves, images and modulation curves delivered to the science data center (SDC) well in advance with respect to the complete scientific and ancillary data. The aim of these data is to monitor the behavior of the source on orbital base.

The design of the ICU is based on the use of double-eurocard boards. The ICU is located inside the Service Module. Currently some ongoing trade-off studies will establish if some of the functionality now allocated into the ICU (and in particular the driver for the FW, that of the Peltier and the DC/DC converters) will be, instead, located in the BEEU. This is due to the current long distance of the ICU from the DU (about $4 \mathrm{~m}$ ).

\section{XIPE IN THE VEGA FAIRING}

We designed XIPE to be installed into the fairing of the Vega launcher with a trade off between volume and mass, therefore performances, and cost. At the time of the proposal we exploited the heritage of POLARIX which telescopes had the same focal length. The Concurrent Design Facility (CDF) of ESA confirmed that indeed Vega is capable to accommodate XIPE. Here we show (see figure 6) the accommodation at the time of the proposal. While writing, the companies which are studying XIPE during the phase A confirmed the accommodation in the Vega launcher (or in the larger Vega C currently under developing) of MMs with even a larger focal length.

\section{SCIENTIFIC PERFORMANCES}

We present here, see table 4, the scientific performances of XIPE based on laboratory measurement on a prototype filled with He-DME mixture at 1-bar. The MDP is (see figure 7) evaluated taking into account the configuration of the proposal with $3.5 \mathrm{~m}$ of focal length and 27 shells. This MDP is actually very conservative due to the increased sensitivity allowed by the $4 \mathrm{~m}, 30$ shell configuration. At the present time the sensitivity is (energy averaged), $10 \%$ better (20\% less needed observing time and $50 \%$ less at higher energies). In the same plot we also showed some representative X-ray sources which are target for X-ray polarimetry.

We show, in table 5 , the number of sources for which is possible to perform sensitive X-ray polarimetry with XIPE. At this regard we assumed a mission lifetime of three years and different threshold (depending on the source class) on the MDP. We used current available X-ray catalogues for deriving the source flux and, therefore, the MDP. We, then, derived the total number of sources with a MDP better then the threshold and the number of source within three years of operation. 


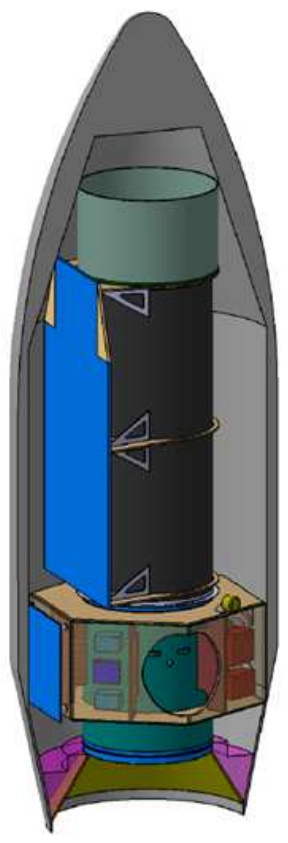

(a)

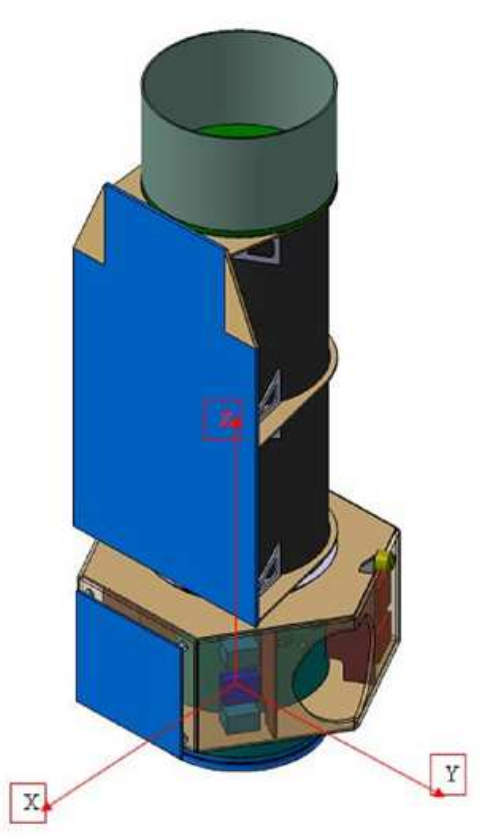

(b)

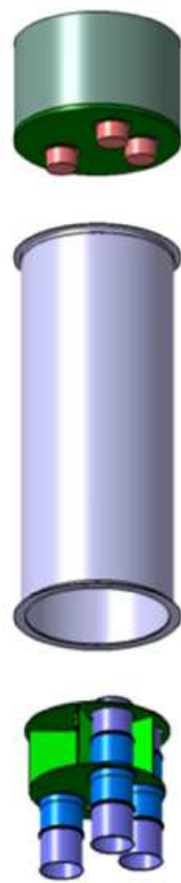

(c)

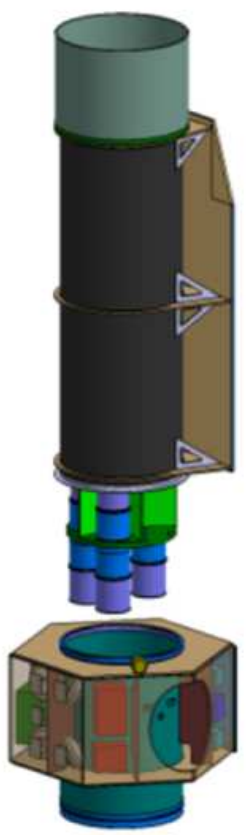

(d)

Figure 6. a. XIPE into the Vega fairing (from the proposal) b. Mirror Modules inside the service module. A Structural Tube connects the Service Module to the Focal Plane Assembly Support Structure c. Three Detector Units and three Back End Electronics Units are disposed with $120^{\circ}$ symmetry onto the focal plane structure. c,d. These figures show how the design allows for a parallel development of the Service Module, the Mirror Modules and the Focal Plane Instrument and the plug\&play philosophy.

Table 4. The characteristics of XIPE.

\begin{tabular}{|c|c|}
\hline Polarization sensitivity & $1.2 \%$ MDP for $2 \times 10^{-10} \mathrm{erg} / \mathrm{s} / \mathrm{cm} 2(10 \mathrm{mCrab})$ in $300 \mathrm{ks}$ \\
\hline Spurious polarization & $<0.5 \%(\mathrm{goal}:<0.1 \%)$ \\
\hline Angular resolution & $<26$ arcsec \\
\hline Field of view. & $15 \times 15 \operatorname{arcmin}^{2}$ \\
\hline Spectral resolution & \begin{tabular}{c}
$16 \% 9 \mathrm{keV}$. \\
\hline Timing
\end{tabular} \\
\hline Resolution $8 \mu \mathrm{s}$ \\
Dead Time $180 \mu \mathrm{s}$ \\
\hline Wall materials & $>3 \mathrm{yr}$ \\
\hline Energy range & Electroformed NiCo \\
\hline Background & $2-8 \mathrm{keV}$ \\
\hline
\end{tabular}

\section{THE CONSORTIUM}

The consortium comprises (see figure 8) many countries across Europe. They have responsibility either in providing payload items but also for work activities related to its development. The responsibility of providing the DU is italian (INFN-Pi) while UK is responsible for the Filter Wheel (UCL/MSSL) and, also, of supervising the thermal-mechanical aspects at system level for the consortium. The High Voltage and Low Voltage Power 


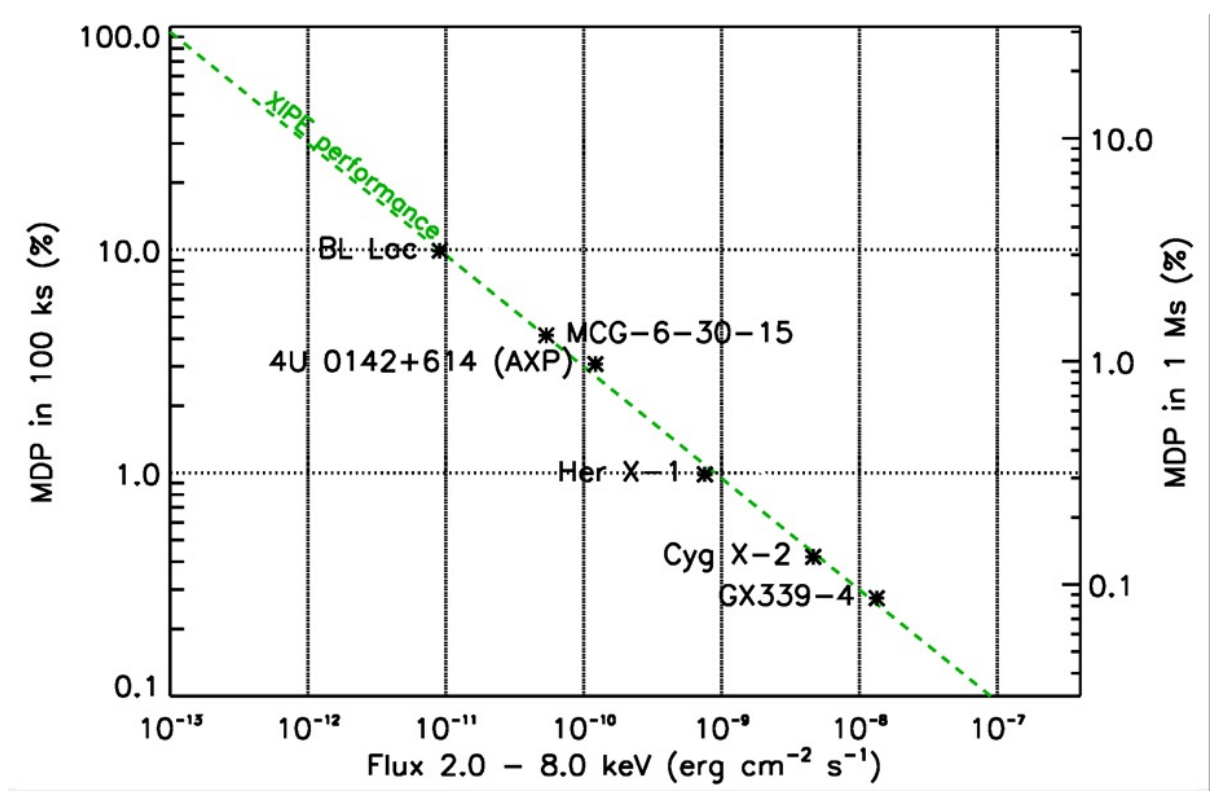

Figure 7. The Minimum Detectable polarization of XIPE taking into account a configuration of $3.5 \mathrm{~m}$ focal length and 27 shells.

Table 5. This table shows how many sources are available to XIPE for performing sensitive polarimetry either during three years of operation and in total. The number of the putative sources are in the range of hundreds and, for each class, a fair number of these can be studied and so not only the brightest ones.

\begin{tabular}{|c|c|c|c|c|c|}
\hline Target Class & $\begin{array}{c}\mathrm{T}_{\text {tot }} \\
(\text { days })\end{array}$ & $\begin{array}{c}\mathrm{T}_{\text {obs }} \\
(\mathrm{Ms})\end{array}$ & $\begin{array}{c}\text { MDP } \\
(\%)\end{array}$ & Number in 3 years & Number available \\
\hline AGN & 219 & 0.3 & $<5$ & 73 & 127 \\
\hline $\begin{array}{c}\text { XRBs } \\
\text { (low+high mass })\end{array}$ & 91 & 0.1 & $<3$ & 8 & 160 \\
\hline SNRe & 80 & 1.0 & $\begin{array}{c}<15 \% \\
(10 \text { regions })\end{array}$ & 6 & 6 \\
\hline PWNe & 30 & 0.5 & $\begin{array}{c}<10 \% \\
\text { (more than 5 regions) }\end{array}$ & 10 & 10 \\
\hline Magnetars & 50. & 0.5 & $\begin{array}{c}<10 \% \\
\text { (more than } 5 \text { bins })\end{array}$ & 2 complexes $(5$ clouds $)$ & 2 complexes $(5$ clouds $)$ \\
\hline Molecular clouds & 30 & $1-2$ & $<10 \%$ & $\mathbf{1 9 3}$ & $\mathbf{3 1 6}$ \\
\hline Total & $\mathbf{5 0 0}$ & & & & \\
\hline
\end{tabular}

Supplies are procured by Poland (SRC/PAS). Germany (IAAT) will provide the ICU, the Electrical Ground Support Equipment (EGSE) and the end-to-end calibration facility (MPE, Panter). Spain (UoV and INTA) is responsible for the FSS and the environmental test. Sweden $(\mathrm{KTH})$ takes care of the evaluation of the background. ESA will procure the X-ray optics, using the valuable expertise of OAB/INAF and it is responsible for the ground segment. Italy (ASI) provides, also, the back-up for Kourou (ESA) of the Ground Station. The Science Data Center is responsibility of Switzerland (UoG). The responsibility of the whole payload is italian (IAPS/INAF). 


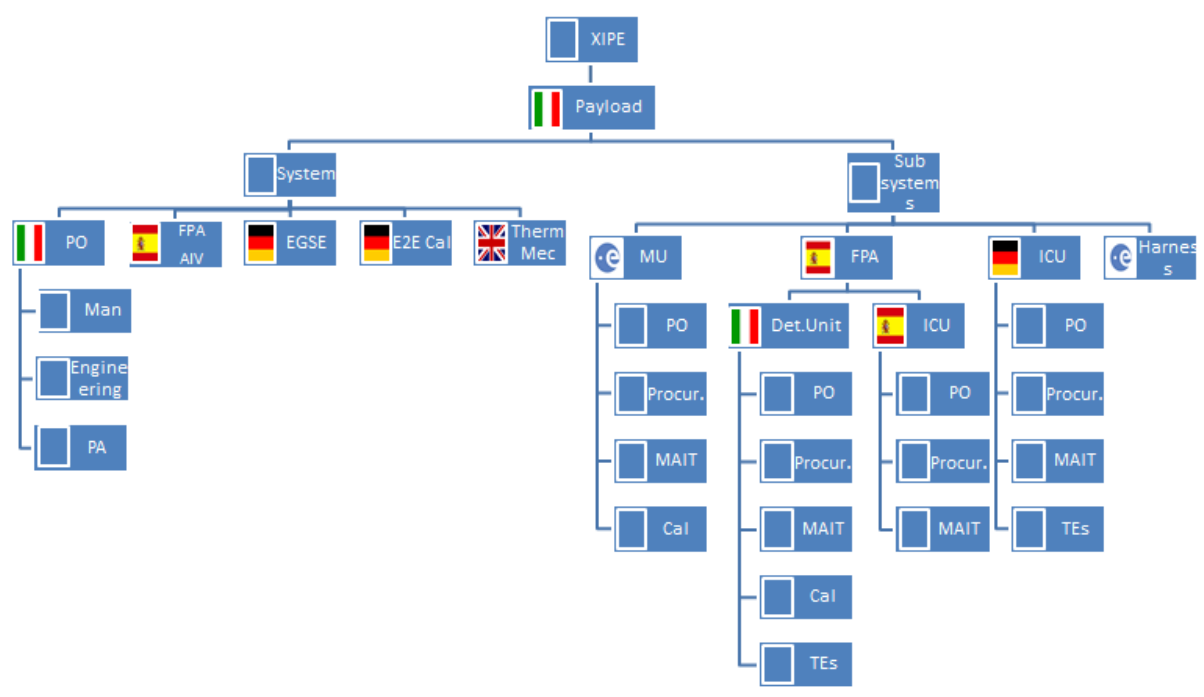

(a)

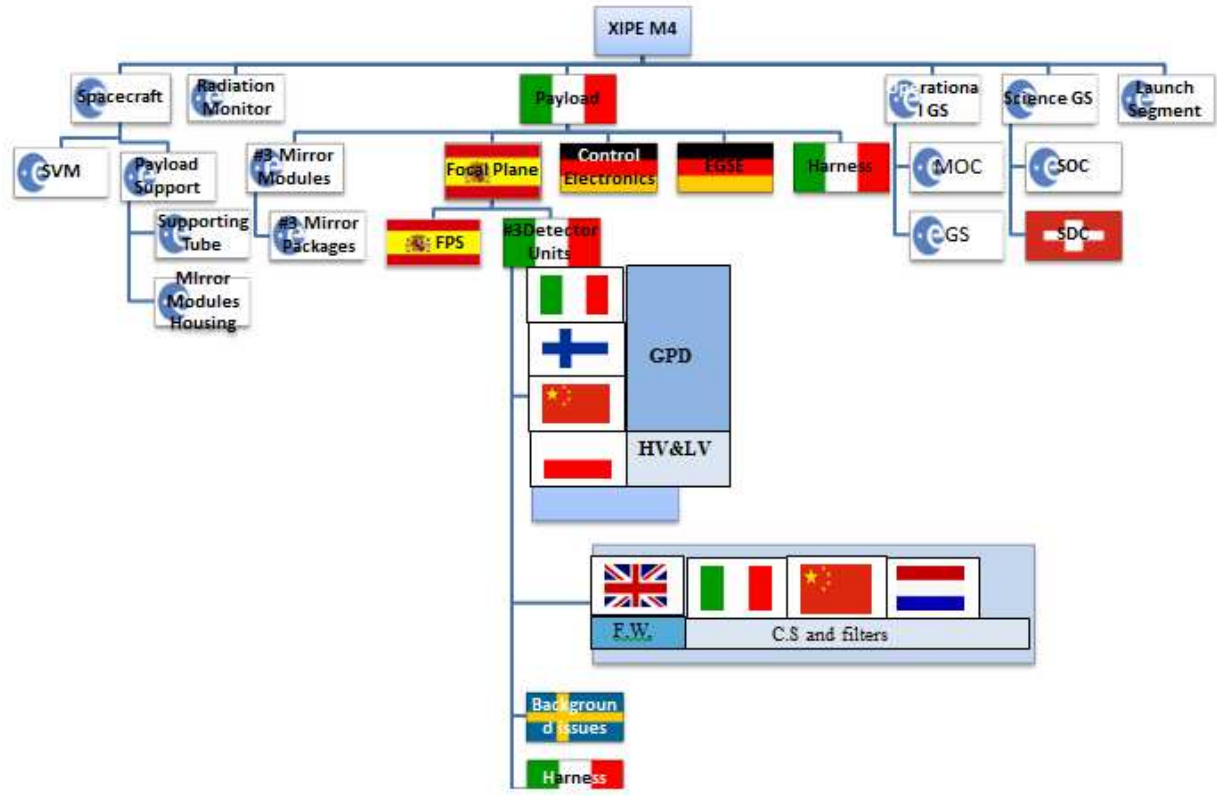

(b)

Figure 8. (a). The Work Breakdown Structure with main national responsibilities(b). The product tree with main and contributory nations. 


\section{ACKNOWLEDGMENTS}

XIPE is developed thanks to the support of ASI(IT), CNSA(CN), DLR (DE), SNPR\&D\&i(ES), SNSB(SE), SRC-PAS(PL), SSO(CH), UKSA(UK).

\section{REFERENCES}

[1] Westfold, K. C., "The Polarization of Synchrotron Radiation.," ApJ 130, 241 (July 1959).

[2] Gnedin, I. N. and Sunyaev, R. A., "Polarization of optical and X-radiation from compact thermal sources with magnetic field," A\&A 36, 379-394 (Dec. 1974).

[3] Rees, M. J., "Expected polarization properties of binary X-ray sources," MNRAS 171, 457 (1975).

[4] Sunyaev, R. A. and Titarchuk, L. G., "Comptonization of low-frequency radiation in accretion disks Angular distribution and polarization of hard radiation," A\&A 143, 374-388 (Feb. 1985).

[5] Mèszàros, P., Novick, R., Szentgyorgyi, A., Chanan, G. A., and Weisskopf, M. C., "Astrophysical implications and observational prospects of X-ray polarimetry," ApJ 324, 1056 (1988).

[6] Gnedin, I. N., Pavlov, G. G., and Shibanov, I. A., "The effect of vacuum birefringence in a magnetic field on the polarization and beaming of X-ray pulsars," Pis ma Astronomicheskii Zhurnal 4, 214 (1978).

[7] Ventura, J., Nagel, W., and Meszaros, P., "Possible vacuum signature in the spectra of X-ray pulsars," ApJ 233, L125 (1979).

[8] Mèszàros, P. and Ventura, J., "Vacuum polarization effects on radiative opacities in a strong magnetic field," Phys. Rev. D 19, 3565 (1979).

[9] Weisskopf, M. C., Elsner, R. F., and O'Dell, S. L., "On understanding the figures of merit for detection and measurement of x-ray polarization," in [Society of Photo-Optical Instrumentation Engineers (SPIE) Conference Series], Society of Photo-Optical Instrumentation Engineers (SPIE) Conference Series 7732 (July 2010).

[10] Weisskopf, M. C., Silver, E. H., Kestenbaum, H. L., Long, K. S., and Novick, R., "A precision measurement of the X-ray polarization of the Crab Nebula without pulsar contamination," ApJ 220, L117 (1978).

[11] Hughes, J. P., Long, K. S., and Novick, R., "A search for X-ray polarization in cosmic X-ray sources," ApJ 280, 255 (1984).

[12] Long, K. S., Chanan, G. A., Ku, W., and Novick, R., "The linear X-ray polarization of Scorpius X-1," ApJ 232, L107-L110 (Sept. 1979).

[13] Costa, E., Soffitta, P., Bellazzini, R., Brez, A., Lumb, N., and Spandre, G., "An efficient photoelectric X-ray polarimeter for the study of black holes and neutron stars," Nature 411, 662 (2001).

[14] Bellazzini, R., Spandre, G., Minuti, M., Baldini, L., Brez, A., Cavalca, F., Latronico, L., Omodei, N., Massai, M. M., Sgro', C., Costa, E., Soffitta, P., Krummenacher, F., and de Oliveira, R., "Direct reading of charge multipliers with a self-triggering CMOS analog chip with $105 \mathrm{k}$ pixels at $50 \mu \mathrm{m}$ pitch," Nuclear Instruments and Methods in Physics Research A 566, 552 (2006).

[15] Bellazzini, R., Spandre, G., Minuti, M., Baldini, L., Brez, A., Latronico, L., Omodei, N., Razzano, M., Massai, M. M., Pesce-Rollins, M., Sgró, C., Costa, E., Soffitta, P., Sipila, H., and Lempinen, E., "A sealed Gas Pixel Detector for X-ray astronomy," Nuclear Instruments and Methods in Physics Research A 579, 853 (2007).

[16] Muleri, F., Soffitta, P., Baldini, L., Bellazzini, R., Brez, A., Costa, E., Di Lalla, N., Del Monte, E., Evangelista, Y., Latronico, L., Manfreda, A., Minuti, M., Pesce-Rollins, M., Pinchera, M., Rubini, A., Sgro', C., Spada, F., and Spandre, G., "Performances of the Gas Pixel Detector: an x-ray imaging polarimeter for upcoming missions of astrophysics," in [Society of Photo-Optical Instrumentation Engineers (SPIE) Conference Series], Society of Photo-Optical Instrumentation Engineers (SPIE) Conference Series (2016).

[17] Soffitta, P., Muleri, F., Fabiani, S., Costa, E., Bellazzini, R., Brez, A., Minuti, M., Pinchera, M., and Spandre, G., "Measurement of the position resolution of the Gas Pixel Detector," Nuclear Instruments and Methods in Physics Research A 700, 99-105 (Feb. 2013).

[18] Fabiani, S., Costa, E., Del Monte, E., Muleri, F., Soffitta, P., Rubini, A., Bellazzini, R., Brez, A., de Ruvo, L., Minuti, M., Pinchera, M., Sgró, C., Spandre, G., Spiga, D., Tagliaferri, G., Pareschi, G., Basso, S., Citterio, O., Burwitz, V., Burkert, W., Menz, B., and Hartner, G., "The Imaging Properties of the Gas Pixel Detector as a Focal Plane Polarimeter," ApJS 212, 25 (June 2014). 
[19] Fabiani, S., Lazzarotto, F., Bellazzini, R., Brez, A., Costa, E., di Cosimo, S., Muleri, F., Rubini, A., Soffitta, P., and Spandre, G., "The Study of PWNe with a photoelectric polarimeter," in [Polarimetry days in Rome: Crab status, theory and prospects], 27 (2008).

[20] Costa, E., Bellazzini, R., Tagliaferri, G., Matt, G., Argan, A., Attinà, P., Baldini, L., Basso, S., Brez, A., Citterio, O., di Cosimo, S., Cotroneo, V., Fabiani, S., Feroci, M., Ferri, A., Latronico, L., Lazzarotto, F., Minuti, M., Morelli, E., Muleri, F., Nicolini, L., Pareschi, G., di Persio, G., Pinchera, M., Razzano, M., Reboa, L., Rubini, A., Salonico, A. M., Sgro', C., Soffitta, P., Spandre, G., Spiga, D., and Trois, A., "POLARIX: a pathfinder mission of X-ray polarimetry," Experimental Astronomy 28, 137-183 (Dec. 2010).

[21] Swank, J. and et al., "Gravity and Extreme Magnetism SMEX (GEMS)," in [X-ray Polarimetry: A New Window in Astrophysics by Ronaldo Bellazzini, Enrico Costa, Giorgio Matt and Gianpiero Tagliaferri. Cambridge University Press, 2010. ISBN: 9780521191845, p. 251], Bellazzini, R., Costa, E., Matt, G., and Tagliaferri, G., eds., 251 (2010).

[22] Jahoda, K., "The Gravity and Extreme Magnetism Small Explorer," in [Society of Photo-Optical Instrumentation Engineers (SPIE) Conference Series], Society of Photo-Optical Instrumentation Engineers (SPIE) Conference Series $\mathbf{7 7 3 2}$ (July 2010).

[23] Black, K., Backer, R. G., Deines-Jones, P., Hill, J. E., and Jahoda, K., "X-ray Polarimetry with a micropattern TPC," Nuclear Instruments and Methods in Physics Research A 551, 755-760 (2007).

[24] Basso, S., Citterio, O., Mazzoleni, F., Pareschi, G., Tagliaferri, G., Valtolina, R., Conconi, P., and Parodi, G., "The problems concerning the integration of very thin mirror shells," in [Optics for EUV, X-Ray, and Gamma-Ray Astronomy IV], Proc. SPIE 7437, 74371C (Aug. 2009).

[25] Burwitz, V., Predehl, P., Friedrich, P., Bräuninger, H., Eder, J., Pfeffermann, E., Burkert, W., Dennerl, K., Hartner, G., Menz, B., Fürmetz, M., Valsecchi, G., Marioni, F., and Grisoni, G., "The calibration and testing of the eROSITA X-ray mirror assemblies," in [Space Telescopes and Instrumentation 2014: Ultraviolet to Gamma Ray], Proc. SPIE 9144, 91441X (July 2014). 